\title{
Petróleo y sumisión: paro petrolero y hegemonía política en Venezuela 2002-2004
}

\author{
Oil and submission: oil unemployment and political hegemony in Venezuela 2002-2004
}

Petróleo e submissão: greve de petróleo e hegemonia política na Venezuela 2002-2004

\section{Luís Fernando Vega}

luisfervega16@gmail.com

https://orcid.org/0000-0003-3927-4677

Universidad del Zulia, Zulia - Venezuela

\section{Luís Guillermo Quintero}

qluisguillermo@gmail.com

https://orcid.org/0000-0002-7819-8753

Universidad del Zulia, Zulia - Venezuela

Artículo recibido en enero 2021 | Arbitraje en febrero 2021 | Aceptación en marzo 2021 | Publicación en abril 2021

\section{RESUMEN}

\section{ABSTRACT}

\section{RESUMO}

El objetivo del presente estudio es analizar el paro petrolero y la hegemonía política de Hugo Chávez desde 2002 hasta el año 2004 a través del control de PETRÓLEOS DE VENEZUELA S.A (PDVSA), hecho que condujo a los ciudadanos del país a una sumisión total, bajo las decisiones del ejecutivo. Esta obediencia tanto de la población, como de los principales líderes opositores nacionales e internacionales ante un liderazgo mesiánico y autoritario estructurado a través de la renta y la propaganda durante el período. La metodología utilizada es descriptiva, con análisis documental. Los resultados de la investigación permiten confirmar que el fenómeno político personalista reactivó el sistema autoritario por encima del modernismo democrático surgido en el siglo XX por los partidos políticos. Además, se constata que el proyecto fue viable por las condiciones de anomia, creadas por el irreconocimiento de la población a la institucionalidad instaurada por el sistema de partidos.

Palabras clave: Petróleo; sumisión; paro petrolero; hegemonía política y Venezuela

The objective of this study is to analyze the oil strike and the political hegemony of Hugo Chávez from 2002 to 2004 through the control of PETRÓLEOS DE VENEZUELA SA (PDVSA), a fact that led the citizens of the country to total submission, under executive decisions. This obedience of both the population and the main national and international opposition leaders before a messianic and authoritarian leadership structured through income and propaganda during the period. The methodology used is descriptive, with documentary analysis. The research results confirm that the personalistic political phenomenon reactivated the authoritarian system above the democratic modernism that emerged in the 20th century from the political parties. In addition, it is verified that the project was viable due to the conditions of anomie, created by the unrecognization of the population to the institutionality established by the party system.

Key words: Petroleum; submission; oil strike; political hegemony and Venezuela

O objetivo deste estudo é analisar a greve de petróleo e a hegemonia política de Hugo Chávez de 2002 a 2004 por meio do controle da PETRÓLEOS DE VENEZUELA SA (PDVSA), fato que levou os cidadãos do país à submissão total, sob as decisões do Executivo. Essa obediência da população e dos principais líderes da oposição nacional e internacional a uma liderança messiânica e autoritária estruturada por meio de aluguel e propaganda durante o período. A metodologia utilizada é descritiva, com análise documental. Os resultados da investigação confirmam que o fenômeno político personalista reativou o sistema autoritário para além do modernismo democrático surgido no século XX pelos partidos políticos. Além disso, verifica-se que o projeto foi viável devido às condições de anomia, geradas pelo não reconhecimento da população da institucionalidade estabelecida pelo sistema partidário.

Palavras-chave: Petróleo; submissão; greve de petróleo; hegemonia política e Venezuela 


\section{INTRODUCCIÓN}

La llegada al poder de Hugo Chávez interrumpe un proceso político consensualista que se había establecido con la caída de la dictadura de Pérez Jiménez, siendo la base de estabilidad construida por los partidos políticos que aceptaban el mecanismo democrático para alternar el poder, y que en efecto lo hicieron durante los 40 años anteriores a 1999. El nuevo gobierno conducido por un ex teniente coronel que había sustituido la vía armada por la electoral en 1997, se plantea un objetivo diferente, desmontar el aparato institucional de partidos para consolidar una hegemonía política, atornillada a una figura personalista encarnada en Hugo Chávez; su principal protagonista.

Para llevar a cabo las pretensiones de la nueva clase gobernante, se inició la construcción de un gobierno autocrático que se iba a extender por todo el Estado, utilizando los mecanismos democráticos, junto a un aparato propagandístico intenso, todo ello sustentado en la renta petrolera, al convertir a Pdvsa en un instrumento político y economía de comando, después del paro petrolero 2002-2003. En este sentido, se pretende analizar el control de Pdvsa a partir del paro petrolero hasta la sumisión total, bajo las decisiones del ejecutivo, presidido por Hugo Chávez, así como la sumisión de la población ante un liderazgo mesiánico y autoritario estructurado a través de la renta y la propaganda durante el periodo 2002-2004.

\section{MÉTODO}

En cuanto a la metodología del presente estudio, fue catalogado como descriptivo, dado que sus objetivos estuvieron orientados a explicar la situación política y económica de Venezuela, basados en los distintos y medios analizados durante los años en que precisó el estudio: desde 2002 a 2004. Ante tales premisas, Arias (2012 p. 24), expresa que "consiste en la caracterización de un hecho, fenómeno, individuo o grupo, con el fin de establecer su estructura o comportamiento". Los resultados de este tipo de investigación se ubican en un nivel intermedio en cuanto a la profundidad de los conocimientos se refiere.

Al mismo tiempo, puede tipificarse estudio como documental, de acuerdo con los planteamientos de Arias (2012, p. 27), se define como "un proceso basado en la búsqueda, recuperación, análisis, crítica e interpretación de datos secundarios, es decir, los obtenidos y registrados por otros investigadores en fuentes documentales: impresas, audiovisuales o electrónicas".

Es por ello que el tipo de investigación se clasifica como documental, dado que la información desarrollada en este estudio, está contenida en fuentes como: leyes, Constitución de la República Bolivariana de Venezuela, periódicos, artículos arbitrados, trabajos de investigación de pregrado y postgrado, entre ellos, se podría destacar: tesis doctorales. En donde para el desarrollo del estudio se clasificó en a). La lógica hegemónica vs autonomía de PDVSA; y b). Autoritarismo y sumisión. 


\section{DESARROLLO Y DISCUSIÓN}

\section{La lógica hegemónica vs autonomía de PDVSA}

Para definir el objetivo rector del gobierno presidido por Hugo Chávez, de crear una hegemonía política, es necesario hilvanar el conjunto de acciones y decisiones que se ejecutan una vez sentados en Miraflores, y su intento de crear instrumentos políticos para alcanzar su meta: permanecer en el poder. En el epígrafe que se presenta a continuación, se intentará dar respuesta a la pregunta de cómo fue el desenlace de los instrumentos hegemónicos impulsado por Hugo Chávez para apoderarse de PDVSA, desestructurando su autonomía, en una dialéctica que genera un ambiente conflictivo en Venezuela.

Por hegemonía, en el sentido que se tratará en todo el ensayo, hace referencia al concepto plasmado por el filósofo italiano Antonio Gramsci (1998) indica que:

se define como el logro de un liderazgo moral, intelectual y político, a través de la expansión de un discurso que fija un significado parcial alrededor de puntos nodales. Involucra más que un consenso pasivo y acciones legítimas: envuelve la expansión de un particular discurso de normas, valores, puntos de vista y percepciones, a través de redescripciones persuasivas del mundo. La lógica de la hegemonía constituye una lógica de la articulación y de la contingencia.

La estructura teórica que plantea el autor es el resultado de un proceso social contemporáneo, es decir, un conjunto de ideas, técnicas e instrumentos sostenido por un ideal común, que pretende imponerse a los demás, y no necesariamente por la coacción material. Es por ello que la lógica interna del gobierno oficialista consiste en tratar de eliminar los contrapesos autónomos que dificulte sus pretensiones hegemónicas, y en el camino se consiguió una tranca que, en su marco racional, era necesaria eliminar: la autonomía corporativa de Pdvsa. "La manera de llevar a cabo ese objetivo la irían dando, pues, las circunstancias.

Así se hizo, a lo largo de un proceso que, marcado por puntos álgidos entrelazados con la polarización política nacional, fue adquiriendo las formas más radicales disponibles" (Urbaneja, 2013, p. 405). Para simplificar lo mencionado por el autor, es fundamental comprender el esquema que motoriza y da movimiento de expansión a los controles hegemónicos impulsados por el chavismo, ese esquema bien definido consiste en:

- Propiciar conflictos

- Obtener victorias

- Acumular poder. 
En primer lugar, por conflictividad se comprende la ausencia de consenso entre grupos sociales que poseen ideas, intereses diferentes y contrarios, y que entre ellos se quiera imponer la voluntad del otro, quien se resiste, creando un clima álgido de tensiones graduales, propensas a aumentar o disminuir según la voluntad de los adversarios. Las condiciones conflictivas aumentaron para el año 2002, los índices de polarización había alcanzado un punto tan tenso que se desencadenó un golpe de estado el 11 de abril.

En retrospectiva, la presión comenzó intensificarse desde el año 2001, al tener una mayoría en la Asamblea Nacional, Hugo Chávez posee un control legislativo que le aprueba todo, en efecto se le concede una ley habilitante que la utiliza para extender el control del gobierno en determinadas materias, con la aprobación de 49 decretos-leyes a finales del 2001, los sectores que ven afectados sus intereses se pronuncian, "la más criticada fue la ley de tierras y la ley de hidrocarburos. Inmediatamente tras esto, los empresarios agrupados en la patronal Fedecámaras, apoyándose de su enorme poder en el país, realizaron una huelga general el 10 de diciembre del 2001, que no logró doblegar a Chávez.

A esta huelga se sumaron los ejecutivos de la empresa estatal petrolera PDVSA deteniendo la producción nacional de crudo" (Prensa Digital Venezolana, 2012). Las medidas tomadas por Hugo Chávez y la movilización de los sectores opositores, agudizan la polarización, al representar cada uno intereses excluyentes, sin puntos de coincidencia que permita llegar a un acuerdo, en consecuencia, las estrategias que utilizaran para dar su siguiente paso será con la intención de eliminar definitivamente a su contrincante, en un clima conflictivo intenso.

En medio de la turbulencia sociopolítica, después que el decreto-ley en materia hidrocarburos concediera todas las competencias petroleras al Ministerio de Energía y Petróleo a finales del 2001, Hugo Chávez nombra en febrero del 2002 una nueva junta directiva presidida por Gastón Parra Luzardo, con la finalidad de sustituir la junta de técnicos profesionales que controlaba Pdvsa, por un equipo controlable bajo la voluntad del ejecutivo, en consecuencia, la medida radicalizó las posturas de los sectores adversos, la alianza entre los opositores presididos por Fedecamaras, Pdvsa, CTV, medios de comunicación, organizaciones y partidos políticos, concentró a los sectores opositores que habían estado fragmentados, lo que "contribuyó al engrosamiento de las filas de la oposición, haciendo converger con los intereses de los poderosos sectores económicos, mediáticos y petroleros, significativas porciones de organizaciones de clase media." (López, 2004).

Las movilizaciones en fechas simbólicas nacionales se hicieron frecuentes en el año 2002, tanto de la oposición como del oficialismo, hasta el punto de radicalizarse la movilización opositora, al exigir la renuncia de Chávez y marchar a Miraflores. El bochorno tuvo lugar en el Puente Llaguno, escenario de 19 muertos por francotiradores, para los opositores se convirtió en la justificación precisa para el golpe de estado, y para el oficialismo en la matriz de opinión para señalar a los culpables. Sin embargo, por esta vía llega los sectores opositores al poder, empero no canalizan bien su triunfo y se voltea la moneda, al regresar Hugo Chávez con el apoyo popular a Miraflores. 
No se pretende abordar de lleno los factores que intervienen en el proceso del golpe de estado, ni cómo fue su desenlace. Sino contextualizar las circunstancias en las que se llevaron a cabo las decisiones y acciones por el control de PDVSA. El aprendizaje del sector oficialista, después del golpe, conllevó a modificar su estrategia, ahora con la pretensión de buscar puntos de coincidencia para poder conciliar con los sectores opositores, la intención era clara, mitigar los índices de conflictividad y mostrar un rostro conciliador ante la población. Sin embargo no hubo posibilidad de acuerdo, ni con la suspensión de la gerencia puesta por Chávez en Pdvsa ni con la intervención de figuras internacionales, ya que las fuerzas opositoras continuaron con su mecanismo insurrecto y excluyente. En diciembre fue el punto más álgido de la paralización económica a nivel nacional:

Este paro mostró connotaciones distintas a los precedentes, al hacerse evidente que no sólo se sumaba el comercio y parte de la industria, o la gerencia administrativa de Pdvsa, como en abril, sino buena parte de la gerencia operativa y los capitanes de la Marina Mercante de Pdvsa. Éstos procedieron en pocos días a paralizar las actividades operativas de la compañía y prácticamente la totalidad de la flota de tanqueros de PDV Marina. El lunes 9 de diciembre, día en que las fuerzas de la oposición convirtieron el paro en «indefinido» hasta que «caiga» Chávez, en cadena oficial trasmitida por todos los medios de comunicación audiovisual, el presidente de Pdvsa, Alí Rodríguez Araque, reconoció que la compañía estaba virtualmente paralizada. En lo que sería un paso decisivo para conjurar la insurrección, Rodríguez Araque denunció el paro como un «sabotaje criminal», convocando al pueblo a salir a la calle y rodear edificios, instalaciones y otros bienes de la empresa, a fin de apoyar al gobierno en su esfuerzo por presionar a la gerencia a levantar la paralización. En las semanas siguientes el pueblo, efectivamente, salió a la calle a rescatar la industria. El conflicto petrolero tomó protagonismo, perdiendo otras acciones y sectores su visibilidad y/o centralidad. (López, 2013).

El impacto sociopolítico y económico que ocasionó el paro petrolero, representó un suicidio político para el sector opositor. Desde el punto de vista mediático, el oficialismo había reorganizado su estrategia, y se mostraba como el pacificador ante la agitación conflictiva que vivía el país, es decir, el conciliador que buscaba remediar la situación ante un adversario que seguía mostrando su rostro violento, al radicalizar las protestas con el paro.

El nuevo vestuario oficialista, ante la fatiga social que ansiaba estabilidad, generó un efecto dominó, al ganar el apoyo popular y acelerar el desgaste de las fuerzas opositoras, y por si fuera poco, crear una matriz de opinión que responsabilizaba a la oposición de la inestabilidad sociopolítica del año 2002 y 2003, justificó su segundo pasó para terminar el trabajo, "culminar con la expulsión de la industria petrolera de unos 20.000 gerentes y empleados, altamente calificados, (...) lo que debió ser uno de los más espectaculares genocidios gerenciales de la historia económica mundial." (Urbaneja 2013, p. 405). 
Con el desgaste de las fuerzas opositoras, el gobierno recluta a Pdvsa, la subordina y la transforma en una economía de comando, es decir, sujeta a las decisiones verticales del poder ejecutivo para cumplir los objetivos políticos de la clase gobernante. Las bases de PDVSA comienzan a cambiar, se sujeta a una nueva política internacional petrolera, basada en la reducción de la producción, que al estar determinadas por la disminución de la oferta con respecto a la demanda, se pretende crear un factor estimulante para el aumento de los precios, directriz que había impulsado Hugo Chávez en su campaña internacional en la Opep. Con ello se desmonta el proyecto de la PDVSA autónoma, cuyo objetivo eran aumentar la producción petrolera para obtener mayor cantidad de ingresos.

Las consecuencias del paro petrolero ocasionó una "devastación económica producida por la paralización de Pdvsa y parte de la industria privada del país tuvo también efectos de debilitamiento de las bases sociales de la oposición, al producirse la quiebra de pequeñas y medianas industrias y comercios, así como un salto de las tasas de desempleo que pasaron del 15,4 por ciento al 20,3 por ciento en cuatro meses. El PIB cayó en el primer trimestre de 2003 a 27 por ciento (Rivas, 2004, citado de López, 2004). Los datos que proporciona el autor se sumarían a los argumentos de la opinión pública oficialista, lo que permitió desprestigiar a las bases opositoras y restarle campo político.

Con ello queda el camino libre para continuar con el proyecto hegemónico, al contar ahora con la mayor riqueza del país a su disposición, se redimensiona las estrategias, y le asigna un valor agregado a los recursos petroleros: el populismo. Si bien es cierto que las practicas populistas habían orientado la administración de la renta en el pasado, ahora con Hugo Chávez controlando totalmente Pdvsa y unos precios petroleros en ascenso, se acentúa el populismo y adquiere formas en las denominadas misiones sociales, que se presentan a la población como "estrategias masivas orientadas a garantizar los derechos fundamentales a la población, con énfasis en los sectores más excluidos.

Estas iniciativas cuentan con recursos extraordinarios, su coordinación es interinstitucional e interministerial y uno de los elementos fundamentales para su planificación, ejecución y seguimiento es la participación activa y protagónica de las comunidades organizadas. PDVSA apoya directamente a casi todas las Misiones Sociales y programas de desarrollo que el Gobierno Bolivariano está impulsando actualmente en el país (PDVSA, 2008, citado de Olivar, 2016, p. 61). La implementación de las misiones sociales se convierten en la táctica fundamental de la estrategia oficialista para ganar el revocatorio, el impacto de estas misiones permitió al liderazgo de Chávez tener mayor contacto con los sectores populares, repuntando su popularidad. "Cuando el 15 de agosto de 2004 se celebró por fin el referéndum revocatorio presidencial, Chávez ya había vuelto a un $45 \%$ de apoyo, ganado con una amplia ventaja a la oposición, con un $59.25 \%$. 
En este sentido, las misiones se fueron ampliando, convirtiéndose en una máquina de multiplicar votos (Cabrices, citado de Vega, 2015, p. 56). El impacto de las misiones fue tan radical en el imaginario sociopolítico de la clase social baja, siendo la mayoría en Venezuela, que todos los candidatos oficialistas a cualquier cargo del poder público en las entidades políticas-territoriales, ofrecían profundizar las misiones, incluso en el sector opositor era un error táctico atacar este régimen de subsidio que profundiza el hacer improductivo, impulsada por un estatismo que aumenta el gasto público al subsidiar una economía parasitaria que la hace depender cada vez más del petróleo.

Dicha dependencia no tiene otro significado sino estar sujeto a la voluntad del gobierno, específicamente del ejecutivo, cuya toma de decisiones y acciones determinarán la vida nacional, sin la intervención de sectores autónomos o contradictorios a la hegemonía política oficialista. La acumulación de poder por el asalto a Pdvsa fue un indicador clave que permite reflexionar hasta qué punto es capaz el gobierno de mantener su proyecto hegemónico, cabe citar tal cual las declaraciones del mismo presidente en el 2004:

"Las crisis muchas veces son necesarias, incluso, a veces hay que generarlas, midiéndolas por supuesto, lo de Pdvsa era necesario, aun cuando nosotros, bueno no es que no la generamos, la generamos-dice bajando el tono de voz- porque cuando yo agarré el micrófono en un aló presidente y empecé a botar gente, yo estaba provocando la crisis, cuando nombre a Gastón Parra Luzardo y aquella nueva junta directiva, estábamos provocando la crisis, ellos respondieron y se presentó el conflicto y aquí estamos hoy-muestra una risa silenciada- era necesaria". (Declaraciones, 15-01-2004).

"Dos elementos claves resaltan en el discurso, la crisis premeditada y medida, y por otro, un gobierno que busca elevar su poder a toda costa. Sacrificando a la población por el bien de la "patria”. En este sentido, ¿Sabía Hugo Chávez en qué terminaría todo el proceso conflictivo entre el 2002 y 2003, jugándose el todo o nada en el intento de apoderarse completamente de PDVSA? Pregunta dejada a la reflexión del lector" (Vega, 2015, p. 55). La estructura del discurso tiene cavidad en el marco de la política estratégica hegemónica, más no en un proyecto democrático y republicano, es decir, la naturaleza del discurso petrolero que usa Hugo Chávez es de carácter conflictivo-proteccionista, que es lo que se quiere trasmitir a la población: mostrar la imagen de un estado capaz de pelear para proteger los "intereses del pueblo".

\section{Autoritarismo y sumisión}

La política petrolera de la llamada Revolución Bolivariana redimensionó el paternalismo estatal, concentrado en la figura de Hugo Chávez, ansiado por el inconsciente colectivo positivista del pueblo venezolano, sobre todo, en tiempos donde el descontento hacia la ineficiencia institucional de la democracia representativa ha dejado un pueblo anómalo, que bajo los efectos patológicos de seguir a hombres fuertes, se dispuso a negociar con un militar para que le resolviera los problemas. 
Para simplificar lo antes mencionado, se partirá explicando que a partir de la crisis interna de la democracia representativa, se desmonta el peso institucional que había mantenido a la sociedad venezolana viviendo en una apariencia cívica y democrática durante los cuarenta años. Los problemas económicos, la corrupción y la poca sintonía de los partidos políticos con los cambios sociales socavaron el terreno institucional y desenterraron la raíz apolítica de la sociedad venezolana, acostumbrada a llamar a hombres fuertes y autoritarios para poner orden a la situación, la imagen y concepto de este fenómeno sociopolítico en el pensamiento colectivo del venezolano forma parte de una síntesis histórica que empezó a gestarse desde la colonia, se acentuó en el siglo XIX con los caudillos y aún manifiesta una plena vigencia en los momentos críticos del sistema político democrático, es por ello que el fenómeno Chávez.

La interrupción del sistema partidista representativo devino por sus propios medios, por las reglas del juego electoral. Una vez convencido Hugo Chávez en 1997 por Luis Miquilena de intentar llegar al poder por la vía electoral, gana las elecciones en 1998, asciende al poder y utiliza los mecanismos institucionales democráticos para desmontar el sistema partidista y desestructurar la estructura republicana, con la finalidad de llevar a cabo una hegemonía política.

Después de obtener el pleno control de PDVSA, su principal fuente de financiamiento para llevar a cabo su proyecto hegemónico, pasa a tener una vinculación más directa con la clase popular, el mensaje que se pretende transmitir es filtrado por un sistema mediático bien estructurado, monopolizado por los medios de comunicación estatal que había aumentado en los primeros años del gobierno, "para 1999 el parque público de medios apenas contaba con la señal de Venezolana de Televisión (VTV), (...) hoy en día, el gobierno en funciones de Estado cuenta en materias de medio radioeléctricos con seis canales de televisión: Venezolana de Televisión, Vive TV (2003), Asamblea Nacional (2005), Ávila TV (2006), Telesur (2005) y Tves (2007) (...) más de ocho emisoras y canales radiales internacionales en convenios con Prensa Latina (Cuba) y la española EFE. (...).

En el sector de las telecomunicaciones cabe mencionar la renacionalización de Cantv, con pleno control en Conatel, Ipostel, Cnti y Redtv" (Bisbal, 2011). El control de los principales sistemas de comunicación masivo del país serán los instrumentos encargados de generar una matriz de opinión en torno a la consigna "ahora Pdvsa es de todos" como discurso petrolero que pretende ensalzar a Chávez como rescatista de Pdvsa del imperio norteamericano y repartidor equitativo de los recursos petroleros.

El mensaje a través del sistema comunicacional estatal fue dirigido a una clase popular cuyas condiciones paupérrimas "durante los años 1997 y 2003 (...) alcanzó el mayor puntaje de las últimas décadas, para el primer semestre del año 1997 el 55,6\% de los hogares se encontraba en condición de pobreza y el $25 \%$ era considerado pobreza extrema; así mismo el repunte del 2003 fue durante el segundo semestre que alcanza el máximo valor, para la fecha el 55,1 \% de los hogares se encontraba en condición de pobreza y el 25,5\% era considerado pobreza extrema, y una tasa de desocupación en 2003 de 19,1\% (Instituto Nacional de Estadística, 2011). 


\section{Discusión}

Al haber realizado el arqueo documental que permitió la reconstrucción del trabajo, basado en la historia reciente en torno al petróleo como instrumento de poder del gobierno del ex presidente de Venezuela, Hugo Chávez es posible aseverar que este rubro se ha constituido como un elemento de notable relevancia para perpetuarse en el poder, no solo en el país, sino también en varios Estados de la región, siendo para esos años diferentes mandatarios con una inclinación de izquierda.

En este sentido, son muchos los esfuerzos llevados a cabo por parte de los investigadores en función de observar una realidad y plasmarla en unas cuantas líneas que permitieran abordar el proceso histórico de hace al menos dos décadas cuando se produjo el paro petrolero, propiciado por los sectores empresariales que demandaban un cambio en las políticas gubernamentales.

Por el contrario, lo que se logró conseguir fue un afianzamiento de las decisiones férreas, ejerciendo el control absoluto de la estatal petrolera, haciendo uso y abuso del poder para lograr esto. Desde ese momento, se verá un líder político con características personalistas más enfáticas que propiciarán un cambio total en la forma de manejar el país, tanto en el aspecto económico como en el social y, principalmente el político.

Con base en ello, las repercusiones tanto para el sector empresarial como para la estatal petrolera tuvieron incidencia inmediata y se debieron adscribir de forma instantánea a lo estipulado por el Ejecutivo o bien, iniciar con los procesos de expropiación y otras decisiones unilaterales que afectaron de forma gradual a un sector que apoyó de manera masiva el paro, también se suman a este análisis los actores de los medios comunicativos radiales y televisivos, los cuales empezaron a ser perseguidos por realizar tal frente.

\section{CONCLUSIONES}

El fenómeno político personalista de Hugo Chávez reactivó el sistema autoritario por encima del modernismo democrático surgido en el siglo xx por los partidos políticos, dicho surgimiento fue producto de la crisis institucional del sistema partidista y su conformación estructural fue desarrollada por el control de Pdvsa, al convertirse en el medio subordinado y primer financista de la llamada "revolución bolivariana".

El proyecto hegemónico que pretendía imponer la clase gobernante solo era posible si se desmontaba el sistema republicano liberal, lo que en efecto ocurrió, a través del control de la renta petrolera que sirvió de atornillador de un liderazgo mesiánico y autoritario que actuaba al margen de la legalidad constitucional, y como único constructor del sistema autoritario que depuraba las autonomías de todo ente que se topaba con su proyecto, tal fue el caso de Pdvsa, que al estar en la zona de influencia de intereses diferentes, se convirtió en el centro de conflicto, cuyos resultaos fue triunfal para el chavismo que destronó su autonomía. 
Además, se constata que el proyecto hegemónico fue viable por las condiciones de anomia, creadas por el irreconocimiento de la población a la institucionalidad instaurada por el sistema de partidos, aunada a la pobreza y desempleo de un elevado sector popular, cuya maquinación estratégica a través de la renta y la propaganda encontró tierra fértil para el germen populista y clientelista que instauró las bases de estabilidad del chavismo, que representó una interrupción al proyecto democrático republicano, y el renacimiento de una visión personificada del poder, siendo el legado caudillista en la historia venezolana.

\section{REFERENCIAS BIBLIOGRÁFICAS}

Arias, F. (2012) “El Proyecto de Investigación. Introducción a la metodología científica". 3era Edición. Caracas-Venezuela. Editorial Episteme

Bisbal, M. (2011). Los medios en Venezuela y la formación de una cultura democrática. ¿Hacia dónde va la comunicación en el país? Artículo de la revista Venezuela: República Democrática. 1era Edición. p. 763-781

Gramsci, A. (1998). Cartas desde la cárcel, Nueva Visión, Bs. As.

López, M. (2004). Venezuela 2001-2004: actores y estrategias. CDC Vol. 21 Nro. 56 Edición agosto 2004. Caracas, Venezuela

López, M. (2013). Venezuela 2001-2004. Actores y estrategias. Cuadernos del Cendes. Vol. 21. N ${ }^{\circ}$ 56. Caracas, Venezuela.
Olivar, N. (2016) Cultura petrolera en Venezuela. Tesis inédita. Universidad del Zulia. Maracaibo, Venezuela

Prensa Digital Venezolana. (2012). Correo del Orinoco. El paro petrolero en Venezuela. Ediciones Digitales.

Urbaneja, D. (2013). La renta y el reclamo. Editorial Alfa. Venezuela

Vega, L. (2015). “Personalismo Político de Hugo Chávez como actor histórico dentro del proceso crítico de la democracia en Venezuela 1992-2013". Trabajo de grado inédito. Facultad de Humanidades y Educación. Universidad del Zulia. Venezuela 